\title{
EDITORIAL
}

\section{QT Interval Prolongation and Beyond}

\author{
Jean-Philippe Couderc, Ph.D., M.B.A.
}

From the Heart Research Follow-up Program, Cardiology, University of Rochester Medical Center, Rochester, New York, NY

Over the past 10 years, the number of publications related to the QT interval increased on a yearly average rate of $10 \%$. Moreover between 1997 and 1999, this average rate was almost doubled (18\%). This increased interest about QT measurement from the scientific community was triggered by findings from the International Registry for the Congenital LQTS and the emerging FDA's concern about drug-induced QT-prolonging problem. The terfenadine case was a milestone. The drug was approved for clinical use in 1985. Eightythree serious cardiac events (including 15 deaths) were reported to the FDA in May 1992. The very same year Seldane became the tenth most prescribed drug in the United States because of its nonsedative antihistaminic properties. In January 1997, the drug was removed from the market and replaced by fexofenadine hydrochloride. During these years, several important publications started questioning the QT interval measurement: its precision, its correction for heart rate dependency, and more recently, the predictive value of its prolongation for cardiac events.

In this issue of the Annals, King et al investigate the errors of the QT measurement in dogs when using multiple heart rate correction formulas: Bazett, Fridericia, Framingham among the most popular ones. The King study demonstrates the superiority of the Van de Water method formula designed in 1989 to correct the QT for heart rate in this animal model. Also, King investigated the individual correction method in which modeling the QT/RR relationship in each animal is required in order to have animal-specific formulas. The error was minimized using this technique, but the error remained equal to or greater than the magnitude of interest when evaluating QT prolongation in drug safety studies.
Similarly in humans, QT dynamicity can be individually modeled relying on Holter recordings that include an average of 90,000 beats. The access to this (amount of) information brings another solution to the problem: controlling rather than correcting QT for heart rate. The Heart Research Follow-up Program's team has developed a software package designed to provide a COMPrehensive Analysis of the repolarization Segment (COMPAS software), and it includes a unique "RR bin" technique for the analysis of QT interval in digital Holter recordings. This method compares QT intervals from single cardiac beats preceded by similar heart rate (RR bin) and thus avoids any bias associated with the use of correction formulas. More importantly, the software has been continuously enhanced during the past 5 years to provide a detailed analysis of the repolarization morphology from surface ECGs. Currently, our incentives are to overcome limitation of the predictive value of QT prolongation as a surrogate marker of cardiac risk in the congenital and acquired form of the LQTS. ${ }^{1}$ In the LQTS registry, $10-15 \%$ of genotyped patients carrying an LQTS-related mutation have a normal QT interval duration. ${ }^{2}$ A significant number of noncardiac drugs with torsadogenic properties are associated with very small changes of the QT interval duration (5 to $10 \mathrm{~ms}$ ). This justifies the need for the development of new markers. Our preliminary research, realized on digital ECG recordings from the FDA warehouse, revealed that there are specific moxifloxacin-induced changes of repolarization morphology in addition to $\mathrm{QT}_{\mathrm{Q}} \mathrm{QT}_{\mathrm{C}}$ prolongation. This $\mathrm{I}_{\mathrm{Kr}}$-inhibitory compound was associated with a reduction in $\mathrm{T}$ wave amplitude, and a reduced repolarization velocity in specific portions of the T loop. The QT interval was also

Address for reprints: Jean-Philippe Couderc, Ph.D., M.B.A., Heart Research Follow-up Program, Box 653, Cardiology, University of Rochester Medical Center, Rochester, NY 14642. Fax: 585-273-5283; E-mail: jean-philippe.couderc@heart.rochester.edu 
significantly prolonged in this data set $(13 \mathrm{~ms})$. Based on a multivariate logistic model, abnormal repolarization morphology was found more relevant than QT prolongation for identifying ECGs from individual on moxifloxacin. Similar ECG abnormalities were evidenced when comparing carriers and noncarriers of a HERG mutation (LQT2 associated with abnormal $\mathrm{I}_{\mathrm{Kr}}$ kinetics). But these abnormalities were not present in LQT1 patients (KCNQ1 mutations associated with abnormal $\mathrm{I}_{\mathrm{Ks}}$ currents). ${ }^{3}$ Thus, these specific morphological abnormalities including lower $\mathrm{T}$ wave amplitude $(\mathrm{mV})$, reduced right and left slope of the $\mathrm{T}$ wave $(\mathrm{mV} / \mathrm{ms})$, and abnormal morphological features of the $\mathrm{T}$ loop are common in the ECG from individuals with reduced $\mathrm{I}_{\mathrm{Kr}}$ kinetics. The computerized quantification of abnormal $\mathrm{T}$ wave morphology and feature associated with HERG mutations and $\mathrm{I}_{\mathrm{Kr}}$-blocking drug, may play an important role in the future assessment of both the identification/penetrance of HERG mutations and the $\mathrm{I}_{\mathrm{Kr}}$ related cardiotoxicity of drugs.

\section{REFERENCES}

1. Couderc JP, Zareba W, Moss AJ. Drug-induced changes of ventricular repolarization: New incentives for quantifying $\mathrm{T}$ wave morphology. Int J Bioelectromagnetism 4; 2002: 167170 .

2. Zareba W, Moss AJ, Schwartz PJ, et al. Influence of genotype on the clinical course of the long-QT syndrome. International Long-QT Syndrome Registry Research Group. N Engl J Med 1998;339:960-965.

3. Couderc JP. Interest of $\mathrm{I}_{\mathrm{kr}}$-related abnormalities of ECGs to improve drug-safety evaluation: Preliminary results from the FDA ECG warehouse and the international registry for the congenital LQTS. Drug Information Association: Challenges and Practical Aspects of Assessing Clinical QT Prolongation/Proaarrhythmia Risk and Implications for the Critical Pathway. Fairmont Hotel, 8 May 2006, Washington, DC: 2006. 